\title{
Cabling Arrangement Influence on Repeatability of Immunity EMC Measurements
}

\author{
J. Hallon, M. Bittera, V. Smieško, K. Kováč \\ Dept. of Measurement, Slovak University of Technology, Ilkovičova 3, 81219 Bratislava, Slovak Republic \\ jozef.hallon@stuba.sk,mikulas.bittera@stuba.sk, viktor.smiesko@stuba.sk,karol.kovac@stuba.sk
}

The paper deals with the analysis of induced current of differential loops of transmission lines during immunity test against electromagnetic field measurement. The influence of geometrical configuration of cabling arrangement on induced current values is surveyed. The problem is described for the case of a short two-wire line creating differential mode loop. The current induced at both ends of the line is the characteristics observed in the performed studies. The comparison between the results of measurements on real physical line, numerical simulations and analytical solution is presented.

Keywords: Electromagnetic compatibility, immunity testing, multiple transmission lines (MTL), influence of electromagnetic field, numerical simulation

\section{INTRODUCTION}

$M$ OST ELECTRICAL devices are connected to external cabling. This ensures power supply, transmission of analogue and digital signals, etc. The cabling is connected with the surrounding environment including electromagnetic (EM) fields. Then it becomes the element of transfer path between the source of disturbance and an electrical device, which becomes subject to disturbance. Hence the present legislation requires the guarantee of immunity against electromagnetic fields of electrical devices available on the market - they have to be tested for immunity. Reproducibility of the testing plays a very important role. During this high frequency immunity test, the configuration of the test place and the testing procedure are the determining elements for the reproducibility of the measurements.

This paper is focused on studying the influence of changes in geometric configuration of cabling upon the repeatability of immunity against EM field tests. It is based upon the analysis of impact of the EM field on short transmission lines. The analysis is carried out by measurements, analytical solution as well as numerical analysis. After understanding the problem by means of simple analytical solutions, we focused mainly on the comparison of the measured and simulated results of currents induced on terminating interfaces of the connection cable models.

\section{THEORETICAL ANALYSIS}

Many authors have published the effects of the EM field on transmission lines. We used, as a base, the theory elaborated by Paul [1], [2]. They have been used and cited by the research community as a base for solving multiple transmission lines (MTL) equations. Their aim is the estimation of currents flowing across impedances at the beginning and the end of the cable.

According to [1], we assumed cabling arrangement consisting of $n$ conductors and one common reference conductor, which is placed in the EM field. From integral Faraday law and electric current continuity equation, one may derive the basic MTL equations:

$$
\begin{aligned}
& \frac{\partial}{\partial z} \mathbf{V}(z, t)+\mathbf{R I}(z, t)+\mathbf{L} \frac{\partial}{\partial t} \mathbf{I}(z, t)=\mathbf{V}_{\mathbf{F}}(z, t) \\
& \frac{\partial}{\partial z} \mathbf{I}(z, t)+\mathbf{G V}(z, t)+\mathbf{C} \frac{\partial}{\partial t} \mathbf{V}(z, t)=\mathbf{I}_{\mathbf{F}}(z, t)
\end{aligned}
$$

where $\mathbf{L}, \mathbf{C}, \mathbf{R}, \mathbf{G}$ are $n \times n$ matrices of per-unit-length inductance $\mathbf{L}$, capacitance $\mathbf{C}$, resistance $\mathbf{R}$ and conductance $\mathbf{G}$ of MTL configuration, matrices $\mathbf{V}(z, t)$ and $\mathbf{I}(z, t)$ are line voltages and currents, right side matrices $\mathbf{V}_{\mathbf{F}}(z, t)$ and $\mathbf{I}_{\mathbf{F}}(z, t)$ contain the effect of the incident field, $t$ is time variable and $z$ is longitudinal coordinate.

$\mathbf{R}$ matrix has elements $\left(r_{i}+r_{0}\right)$ in its diagonal, all other elements have the value of $r_{0}$, where $r_{0}$ is the resistance of common conductor and $r_{i}$ are resistances of separate wires. Similarly, in $\mathbf{L}$ matrix $l_{i i}$ elements are intrinsic inductances of separate wires and $l_{i j}$ constitute mutual inductances between $i$-th and $j$-th wire. In $\mathbf{C}$ matrix $c_{i i}$ elements correspond to capacity between $i$-th and common wire and $c_{i j}$ constitute negative capacities between $i$-th and $j$-th wire. Finally, in $\mathbf{G}$ matrix $g_{i i}$ elements correspond to conductivity between $i$-th and common wire and $g_{i j}$ constitute negative conductivities between $i$-th and $j$-th wire.

Using Thevenin's theorem, we can express voltages at the beginning and the end of transmission line by formulas:

$$
\begin{aligned}
& \mathbf{V}(0, t)=-\mathbf{Z}_{\mathbf{S}} \cdot \mathbf{I}(0, t) \\
& \mathbf{V}(\mathscr{L}, t)=\mathbf{Z}_{\mathbf{L}} \cdot \mathbf{I}(\mathscr{L}, t)
\end{aligned}
$$

where $\mathbf{Z}_{\mathbf{S}}$ and $\mathbf{Z}_{\mathbf{L}}$ are matrices of input and output resistances (general impedances), 0 represents the beginning and $\mathscr{L}$ the end of MTL.

For simplification in further analysis, we can assume a two-wire symmetrical line loaded by terminating impedances $Z_{S}$ and $Z_{L}$ (Fig.1) placed in homogeneous space. Then we can assume line excitement by normalized plane EM wave. From the general MTL model we can switch to pair line, where matrices become complex scalar variables. By this way we obtain sufficiently simple mathematical expressions. 


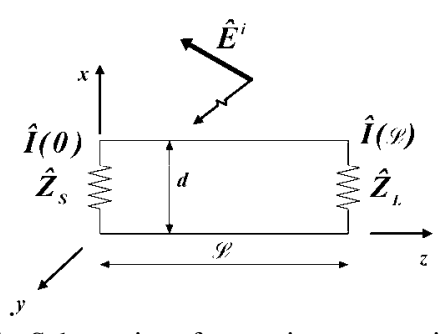

Fig.1 Schematics of two-wire symmetrical line.

The relation for the current at the beginning of two wire symmetrical line load by impedances $Z_{S}$ and $Z_{L}$ (Fig.1) is:

$$
\begin{aligned}
& \hat{I}(0)=\frac{d \hat{E}_{0}}{\hat{D}} e^{-j \beta_{x} d / 2}\left[\frac{\sin \left(\frac{\beta_{x} d}{2}\right)}{\left.\frac{\beta_{x} d}{2}\right] \times}\right. \\
& \times\left\{-j \beta_{x} e_{z} \int_{0}^{\mathscr{2}}\left[\cosh (\hat{\gamma}(\mathscr{D}-\tau))+\sinh (\hat{\gamma}(\mathscr{D}-\tau)) \frac{\hat{Z}_{L}}{\hat{Z}_{C}}\right] e^{-j \beta_{z} \tau} d \tau+\right. \\
& \left.+e_{x}\left[\cosh (\hat{\gamma} \mathscr{D})+\sinh (\hat{\gamma} \mathscr{D}) \frac{\hat{Z}_{L}}{\hat{Z}_{C}}-e^{-j \beta_{z} \mathscr{L}}\right]\right\}
\end{aligned}
$$

where

$$
\hat{D}=\cos (\hat{\gamma} \mathscr{L})\left(\hat{Z}_{S}+\hat{Z}_{L}\right)+j \sin (\hat{\gamma} \mathscr{\mathscr { L }})\left(\hat{Z}_{C}+\frac{\hat{Z}_{S} \hat{Z}_{L}}{\hat{Z}_{C}}\right)
$$

The cross-section of the line lies in $x y$-plane and the length $\mathscr{L}$ is in the direction of $z$-axis. The exciting field is constituted by a normalized EM plane wave represented by intensity $E_{0}$ and three corresponding angles or by the corresponding field intensity components $e_{x}, e_{y}, e_{z}$ respectively. $\beta$ is phase constant $\beta=\omega \sqrt{\mu \varepsilon}$, while $\beta_{x}, \beta_{y}, \beta_{z}$ are its corresponding components and $Z_{c}$ is characteristic impedance of the line.

To further simplify the problem, we assumed only a lossfree line $(R=G=0)$ and a horizontally polarized plane wave propagating in the plane of transmission line. Then the corresponding angles change:

$$
\theta_{E}=0^{\circ}, \quad \theta_{P}=180^{\circ}, \quad \phi_{P}=0^{\circ} .
$$

It means that the components of the vector of electrical field strength $E_{i}$ and phase constant $\beta$ along coordinate axis are as follows:

$$
e_{x}=0, \quad e_{y}=0, \quad e_{z}=1, \quad \beta_{x}=\beta, \quad \beta_{y}=0, \quad \beta_{z}=0
$$

Using the substitution $\hat{\gamma}=\mathrm{j} \beta, \quad \cosh x=\cos j x$, $\sinh x=-j \sin j x$ we obtain a simplified formula:

$$
\hat{I}(0)=\frac{d \hat{E}_{0}}{\hat{D}} e^{-j \beta d / 2}\left[\frac{\sin \left(\frac{\beta d}{2}\right)}{\frac{\beta d}{2}}\right] \times\left[\frac{\hat{Z}_{L}}{\hat{Z}_{C}}(\cos (\beta \mathscr{D})-1)+j \sin (\beta \mathscr{D})\right]
$$

where

$$
\hat{D}=\cos (\beta \mathscr{D})\left(\hat{Z}_{S}+\hat{Z}_{L}\right)+j \sin (\beta \mathscr{D})\left(\hat{Z}_{C}+\frac{\hat{Z}_{S} \hat{Z}_{L}}{\hat{Z}_{C}}\right)
$$

$Z_{c}$ is characteristic impedance of loss-free line

$$
Z_{C}=\sqrt{\frac{l}{c}}
$$

$l$ and $c$ are per-unit length inductance or capacitance of the line

$$
\begin{gathered}
l=\frac{\mu}{2 \pi} \ln \left(\frac{d^{2}}{r}\right) \\
c=\frac{2 \pi \varepsilon}{\ln \left(\frac{d^{2}}{2 r}\right)}
\end{gathered}
$$

$\mathscr{L}$ is the length of the line, $d$ the distance between wires in $\mathrm{x}$ axis direction, $r$ radius of the wires and $\mu$ and $\varepsilon$ permeability and permittivity of the medium.

Then equation (5) can be used to analyze the induced currents through the transmission line.

\section{NUMERICAL MODEL}

It is evident that the presented theory is applicable first of all for simple examples of MTL in a homogeneous space, which are excited by a normalized plane EM wave. To achieve a reasonably simple mathematical formulation in the case of other MTL, further simplifications must be used. However, in the case of MTL with a more complicated shape in nonhomogeneous space, the analytical solution can cause significant errors, e.g. measuring a wooden table, on which the tested equipment with its cabling is situated, can be mentioned. The presence of the table influences the distribution of the EM field in its surrounding by changing the velocity of propagation of the EM wave in the environment, which has material parameters different from free space, and by additional reflections at the boundary of a table and free space.

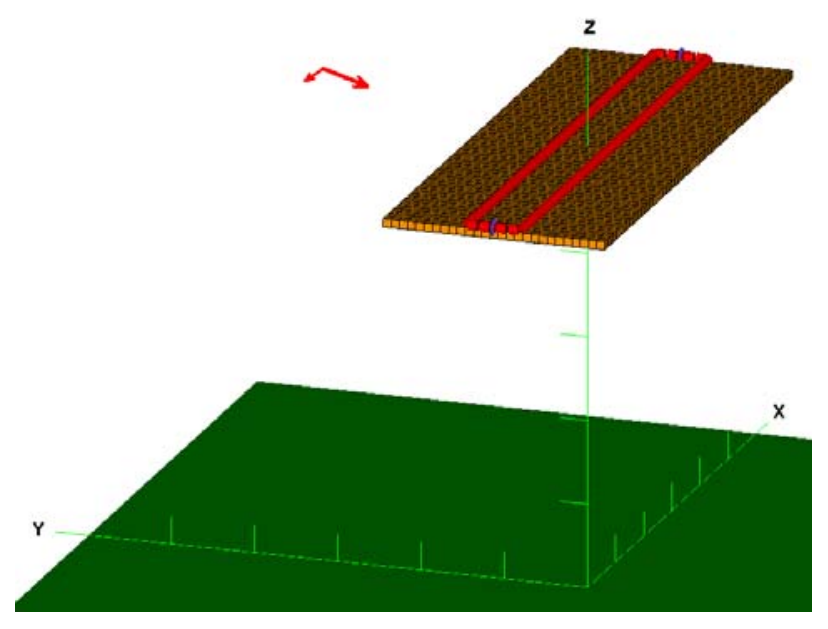

Fig.2 Simulation model of analyzed differential loop. 
It also affects the amplitude of induced current through MTL and its impedances and shape of its frequency dependence [3], [4]. For this reason it is more suitable to use numerical simulators to find out the MTL solution. For wire geometries in combination with 3D objects the simulator based on the Method of Moments is suitable. Therefore, the numerical simulator FEKO was chosen.

The numerical model of differential mode transmission line loop is shown in Fig.2. The conducting loop consists of a larger number of segments. At the ends of the line, there are impedances with defined values and dimensions according to the real loop. This model allows us to change the dimensions of the loop as well as its position easily, as desired. It also allows changing both loading impedances independently, as each may be established by a serial combination of electrical resistance, capacitance and inductance. In predefined distance under the loop, a dielectric board, which constitutes the supporting measurement table, may be placed. Also, a perfectly conductive plane corresponding to the conducting floor of the measuring place is situated in a defined distance. The source of excitation was specified as a linearly polarized plane wave with a given intensity, polarization (horizontal or vertical) and direction of propagation. The last parameter was chosen to estimate the incident wave from the transmitting antenna placed in $3 \mathrm{~m}$ distance and height of $1.55 \mathrm{~m}$. It is because such situation corresponds to the real configuration during EMC immunity tests (Fig.3). During the numerical simulation, the current induced in differential loop in the point of terminating impedance $Z_{L}$ is monitored.

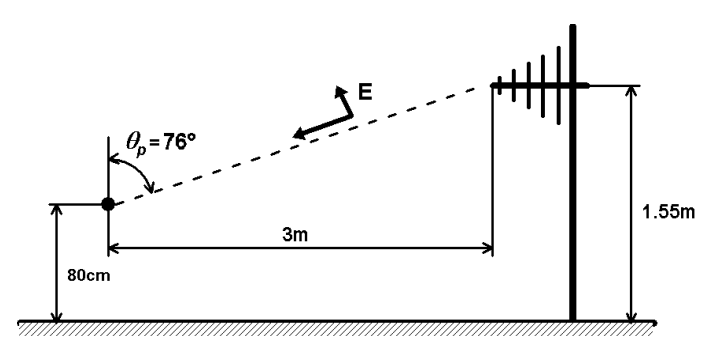

Fig.3 Geometrical arrangement of testing workplace.

\section{MEASUREMENT SETUP}

Usually the differential RF currents are measured by current probes [5], [6]. But in our experiment this is not appropriate because the probe with output coaxial cable becomes a part of the measuring object arrangement. Moreover, the conducting cover of the probe and its cable change the field homogeneity in its surroundings. This fact has an evident influence upon the measurement repeatability, especially in the case of the examined short lines. The operating range of current probes is limited by their use in up to hundreds of $\mathrm{MHz}$. Also, these probes are frequency dependent and they have low sensitivity.

However, it is necessary to measure the current through the line with minimal influence on the measured quantity. Due to the absence of reference potential along the differential loop, it was necessary to build a fully symmetrical measuring circuit (see Fig.4). Only by using this configuration we could obtain the differential component of induced current in the loop. This means that the measuring equipment should have minimal size and no feed cable, which can create parasitic couplings. Therefore, it was necessary to use internal power supply for the probe and data transmission via optical cable. The measured two-wire transmission line was terminated by the impedance of input attenuator of the measuring equipment. This attenuator ensures also RF isolation between the line and detector and reduces mismatch of the circuit. Sigma-delta AD converter converts output signal of the detector into digital form. Then the measured data, processed by a micro-controller and optical converter, are transmitted out of the shielding room via fibre optic.

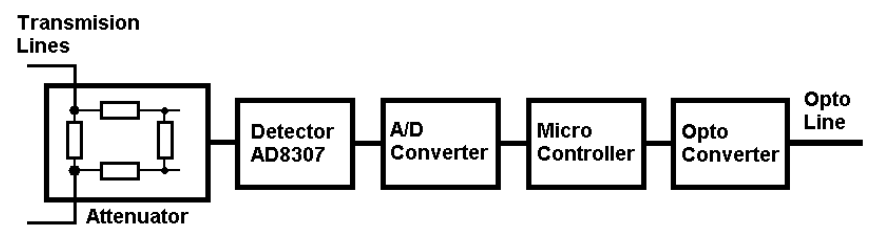

Fig.4 Block diagram of measuring equipment.

An inevitable requirement of the measuring equipment is its symmetry. Therefore, it is not possible to design the detector as a serial diode detector with Schottky diode. Asymmetry of such a circuit causes that in spite of common mode signal at its input there is differential voltage at its output. For this reason, we had to equip our measuring circuit with AD9307 logarithmic amplifier [7]. This circuit has a fully symmetrical input, frequency range from DC up to $500 \mathrm{MHz}$ (with lower linearity even up to $1 \mathrm{GHz}$ ) and dynamical range of $92 \mathrm{~dB}$. A typical application of the detector AD8307 is shown in Fig.5.

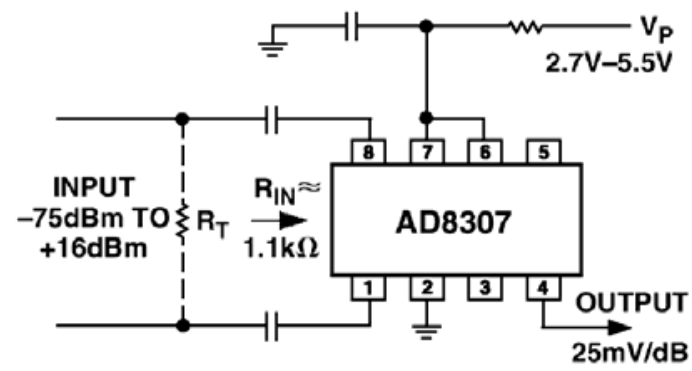

Fig.5 Typical application of detector AD8307.

A testing place was created and calibrated according to international standard [8]. Also, the measuring procedure was identical to the procedure described in this standard. The intensity of the electric part of EM field was $10 \mathrm{~V} / \mathrm{m}$ at $(-0$, $+6 \mathrm{~dB}$ ) each frequency. The frequency was changed gradually with $1 \%$ step within the frequency range from $80 \mathrm{MHz}$ to $1 \mathrm{GHz}$. However, we measured just up to $480 \mathrm{MHz}$, because just this frequency range was covered with sufficient power of 
the radiated field. Our long-time experience in the field of EMC testing also indicates that the majority of tested device failures are in this frequency range. The transmitting logperiodic antenna was placed at the height of $155 \mathrm{~cm}$ above the reference plane in $3 \mathrm{~m}$ measuring distance (Fig.3). The results presented further were measured under horizontal polarization of the transmitting antenna.

\section{RESULTS AND DISCUSSION}

First, the behaviour of the difference loop was surveyed based on analytical solution of induced current (5) in the loop. Induced current at terminating impedance $50 \Omega$ was computed while we assumed that the transmission line is situated in a constant EM field of $10 \mathrm{~V} / \mathrm{m}$. Two analyses were performed:

- Dependence of induced current on changing length of transmission line $(\mathscr{L}=1 \div 3 \mathrm{~m})$ with constant distance between wires $d=1 \mathrm{~cm}$;

- Dependence of induced current on distance between wires $(d=1 \div 25 \mathrm{~cm})$ with constant length of transmission line.

In both cases we assumed wires with radius of $0.5 \mathrm{~mm}$. The current was computed for the frequency range $80 \div 1000 \mathrm{MHz}$. Terminating impedances are identical at both ends of the transmission line, so based on symmetry of the line induced currents are also identical at both ends.

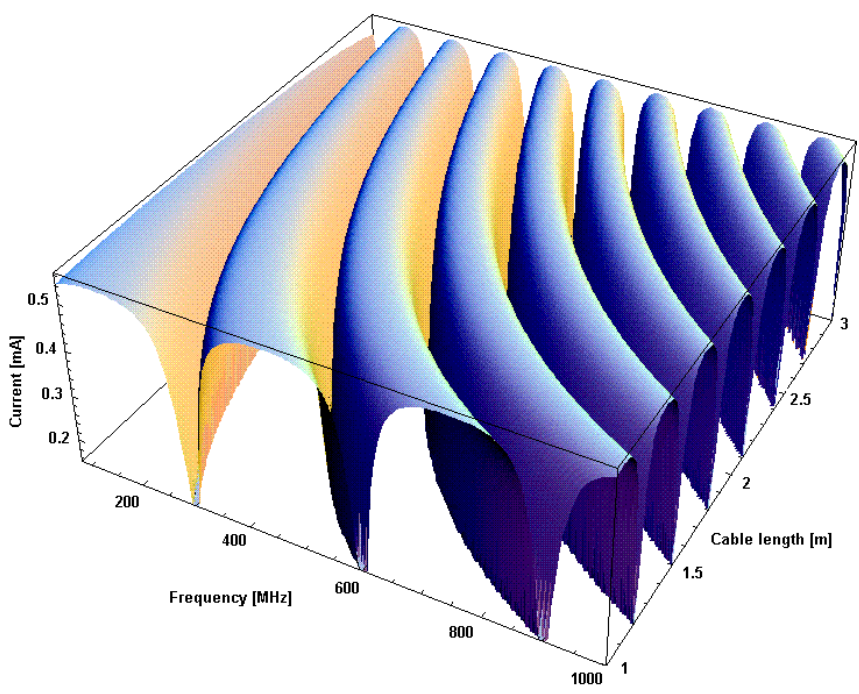

Fig.6 Frequency dependence of induced current on $50 \Omega$ terminating impedance of the transmission line for long lines $1 \div 3 \mathrm{~m}$.

The results of the analysis are shown in Fig.6 and 7. It is evident that with the increasing length of transmission line the extremes (minimums and maximums) of frequency dependences of induced current are moving into lower frequencies. Then, at the same frequency range we get more extremes. From the analysis we can see:

- Minimums of current are at frequencies: $f_{n}=n c / \mathscr{L}$,

- Maximum of current are at frequencies: $f_{m}=(2 m+1) c l$ $2 \mathscr{L}$ where $n$ a $m$ are natural numbers and $c$ is light speed in free space. The values of induced current maximums are equal for the entire analysis. On the other hand, current maximums change their value if we change the distance between wires, so we change the loop area. In Fig.7 we can see the increase of induced current with raising width of the transmission line with the length $\mathscr{L}=2 \mathrm{~m}$. Its behaviour is given by the ratio of distance $d$ and wavelength of the incident field. While the increase of the current is linear with raising distance $d$ at lower frequencies, at higher frequencies it is not so clear - it is part of a sinusoid. As it was shown in the previous analysis, the length of the cable influences the number of current extremes in the given frequency range.

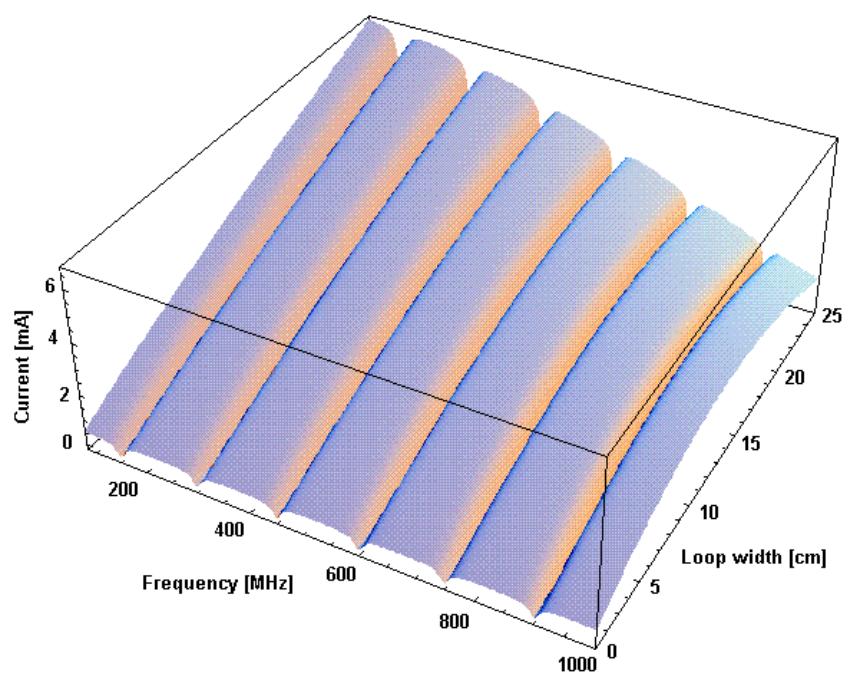

Fig.7 Frequency dependence of induced current on $50 \Omega$ terminating impedance of transmission line for loop width $1 \div 25 \mathrm{~cm}$.

Because of many simplifications in the previous analysis, it was inconceivable to include also the influence of reference ground and non-conducting table. However, their effect cannot be abandoned, so we performed other analyses using numerical simulations. Then the same real measurements were carried out to compare the results.

We focused our attention to similar problems that were solved in the previous analysis. Increasing the length of transmission lines causes more extremes of induced current at the frequency range of our interest similarly to the previous analysis. On the other hand, the shape of current frequency dependence between two minimums is different. It is not as symmetrical as in the theoretical assumption, the maximum is more sharp-edged and it is moved close to the following minimum. This non-symmetrical shape is influenced especially by the presence of reference ground $80 \mathrm{~cm}$ under the differential loop of transmission line. Non-conducting table affects the position of induced current minimums in the frequency range. Unfortunately, it is impossible to perform such measurements because of limited size of the test site.

Next, an analysis of the influence of the width of differential mode loop was performed for $1 \mathrm{~m}$ long transmission lines placed in $80 \mathrm{~cm}$ height above the reference plane. The width of the loop was varied by means of changing 
distance between wires $d$. In Fig. 8 there are shown simulated and measured frequency dependences of induced voltages for four loop widths. It is evident that this parameter has major influence on the amplitude of induced current. Hence it is apparent that the cables of each circuit have to be placed in close proximity to each other. The position of minimum of current within frequency range $230-280 \mathrm{MHz}$ depends mainly on the length transmission lines, but it is affected also by distance $d$.
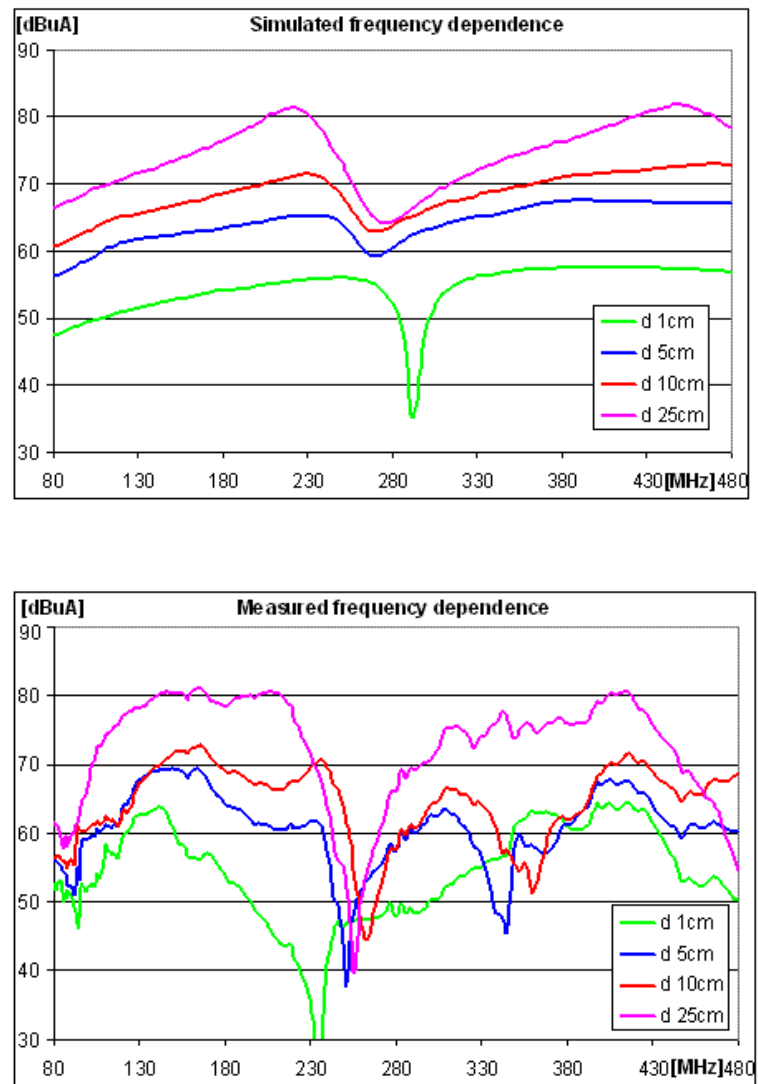

Fig.8 Simulated and measured frequency dependence of induced current through $50 \Omega$ terminating impedance of differential mode loop for four loop width $-1,5,10$ and $25 \mathrm{~cm}$.

Moreover, the analysis of the influence of the additional shielding plane of differential loop was performed for $1 \mathrm{~m}$ long lines with $10 \mathrm{~cm}$ wide loop. This experiment shows the effect of a grounded shielding plate placed near short lines. The shielding plate is created by $1.5 \mathrm{~mm}$ aluminum metal sheet located in the height of $80 \mathrm{~cm}$ over reference ground with the same potential as the ground plane. In Fig.9 are shown simulated and measured results for four different heights of the loop. In spite of small differences between configurations of the simulation and measurement, obtained spectra have similar tendencies. These results show the importance of a grounded shielded plate placed near the lines to achieve sufficient immunity level of electrical devices.
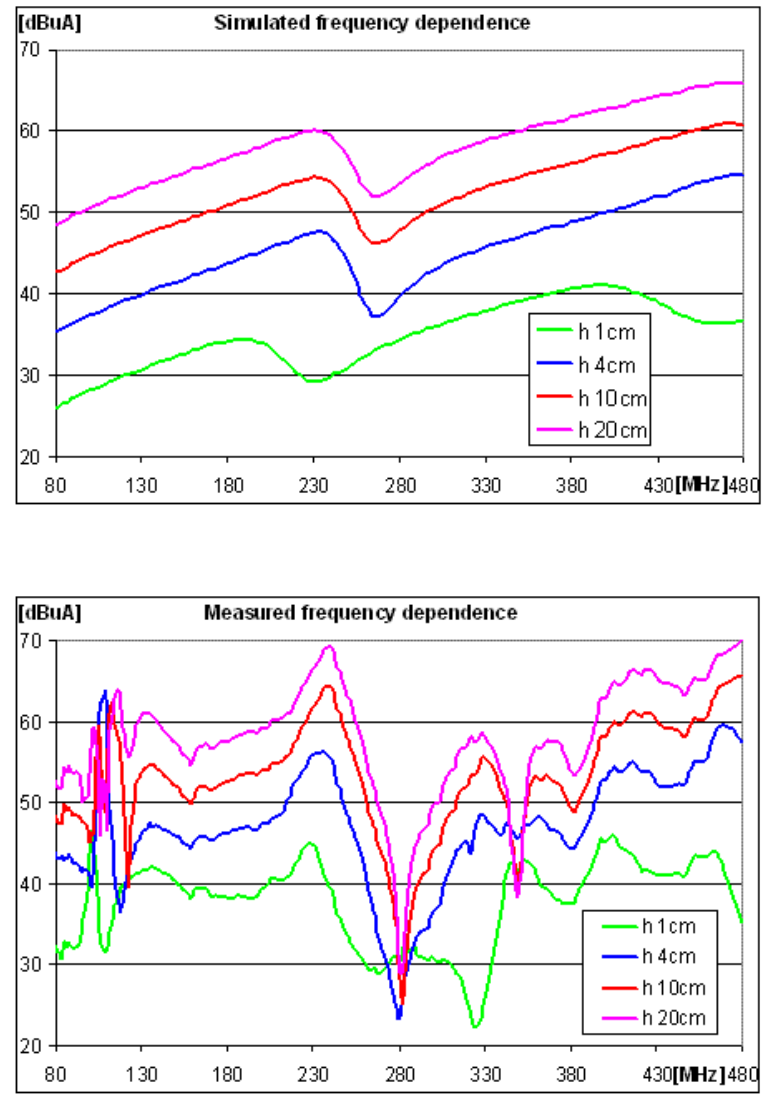

Fig.9 Simulated and measured frequency dependence of induced current through $50 \Omega$ terminating impedance of differential mode loop for four lines heights.

\section{CONCLUSIONS}

By using analytical solution, the behaviour of induced differential current in a transmission line was obtained. This approach unfortunately does not include important additional effects, such as presence of reference ground, etc.

The designed measuring technique for differential current measurements was approved as a suitable tool to verify the models of coupling electromagnetic field to connecting cables, modelled as MTL. By using this tool it was possible to study the behaviour of MTL and its sensitivity to changes of MTL geometry.

As the above analysis shows, it is suitable to place wires of a connecting line at close quarters, if possible. On the other hand, the single lines should be placed separately not to influence themselves mutually. It is also possible to use shielding techniques to RF separation to increase susceptibility against disturbing EM field.

\section{ACKNOWLEDGMENT}

The work presented in this paper was supported by the Slovak Ministry of Education under grant No. 2003SP200280802 and by the Slovak Grant Agency VEGA under grant No. VEGA 1/3101/06. 


\section{REFERENCES}

[1] Paul, C.R. (1994). Analysis of multiconductor transmission lines. New York, USA: Wiley Interscience.

[2] Paul, C.R. (1994). A SPICE model for multiconductor transmission lines excited by an incident electromagnetic field. IEEE Transactions on Electromagnetic Compatibility 36(4), 342 - 354.

[3] Beggio, A., Bono, G., Zich, R.E. (1999). Analysis of the unwanted effects on the radiated emissions and susceptibility measurements due to the introduction of a wooden table. In IEEE EMC Symposium 1999, 2-6 August 1999 (pp. 761-764). Seattle, USA.

[4] Beeckman, P.A. (2001). The influence of positioning tables on the results of radiated EMC measurements. In IEEE EMC Symposium 2001, 13-17 August 2001 (pp. 280-285). Montreal, Canada.
[5] Omid, M., Kami, Y., Hayakawa, M. (1997). Field coupling to nonuniform and uniform transmission lines. IEEE Transactions on Electromagnetic Compatibility 39(3), $201-211$.

[6] Adams, J. W., Cruz, J., Melquist, D. (1992). Comparison measurements of currents induced by radiation and injection. IEEE Transactions on Electromagnetic Compatibility 34(3), 360 -362.

[7] ANALOG DEVICES, Inc. (2003) Low cost DC-500 $\mathrm{MHz}, 92 \mathrm{~dB}$ logarithmic amplifier AD8307. Retrieved November 2003, from http://www.analog.com

[8] Electromagnetic compatibility (EMC) - part 4-3: testing and measurement techniques - radiated, radio-frequency, electromagnetic field immunity test. (1996). Slovak version of European standard. STN EN 61000-4-3. 